\title{
Prometheus revisited
}

\author{
Kai-Yuan Chen, Xiling Shen, and Anna Mae Diehl
}

Center for Cenomics and Computational Biology, Department of Biomedical Engineering and Division of Gastroenterology, Department of Medicine, Duke University, Durham, North Carolina, USA

The liver's extraordinary ability to regenerate has been known since the myth of Prometheus, but the mechanisms involved are still being discovered. Various small animal models have been used in this quest. Two of the most popular include partial hepatectomy (PHx), in which two-thirds of the liver mass is surgically removed to evoke a massive, immediate stimulus for regeneration, and prolonged exposure to toxins that kill liver cells more gradually, provoking chronic regenerative activity. In either case, multiple types of cells must interact effectively to repopulate the organ with functional mature hepatocytes and thus assure ultimate restoration of healthy liver structure and function. This complexity has confounded efforts to distinguish specific changes that occur in cells that repopulate the hepatocyte compartment from changes in other cell populations, including subpopulations of hepatocytes or hepatocyte precursors that do not become regenerative. In the current issue of the $J \mathrm{Cl}$, Wang et al. used translating ribosome affinity purification followed by high-throughput RNA sequencing (TRAP-seq) to isolate mRNAs from repopulating hepatocytes in order to profile gene expression specifically in the hepatocytes that regenerate the liver following toxic injury imposed by inherent byproducts of tyrosine metabolism. This innovative methodology can potentially be used to design therapeutic strategies for liver regeneration.

\section{Liver regeneration}

The liver plays an essential role in various processes such as metabolism, drug detoxification, optimal functioning of the brain, and secretion of bile. Most of these vital liver-specific functions are carried out by parenchymal cells called hepatocytes. These cells rarely divide in healthy adult livers. However, after liver damage, some of the healthy surviving hepatocytes become proliferative; others that sustained damage are acutely inhibited from completing the cell cycle; some of these become senescent permanently and eventually die. Because health requires a sufficient mass of mature hepatocytes to accomplish vital liver-specific functions, repopulating the mature hepatocyte compartment is a regenerative priority. When replication of mature hepatocytes is not sufficient to accomplish this, hepatic progenitors are mobilized. The cellular origin of hepatocyte progenitors is controversial and may vary according to the type, magnitude, and duration of liver injury. Dedifferentiated hepatocytes, bile duct cells (cholangiocytes), bipotent hepatoblasts that can differentiate into either hepatocytes or cholangiocytes, and liver-resident (or bone marrow-derived) multipotent mesenchymal stem-like cells are all capable of generating hepatocytes experimentally. Although they vary in their ability to perform or acquire functions of mature hepatocytes, each of these cell types also produces mitogens and various factors that modulate tissue inflammation and local remodeling of the vasculature and

Related Article: p. 2297

Conflict of interest: The authors have declared that no conflict of interest exists

Reference information: / Clin Invest. 2018;128(6):2192-2193. https://doi.org/10.1172/JCI120933.

matrix. Dysregulation of these processes results in defective regenerative responses that promote acute liver failure and liver cancer, and thus preventing and treating these life-threatening diseases will be expedited by delineating the mechanisms for effective liver regeneration.

\section{Novel method to label regenerating cells}

In this issue of the JCI, Wang et al. introduce a novel approach - translating ribosome affinity purification followed by RNA sequencing (TRAP-seq) - to profile ribosome-associated translating messenger RNAs of replicating hepatocytes (1). By coupling this technology with hepatocyte-selective "marking" strategies in mouse models, the authors overcome the typical confounding issue of contamination by other cell types and thus provide a highly specific characterization of gene products that are differentially up- or downregulated in proliferative versus quiescent hepatocytes. As mentioned above, the results have potential clinical relevance to scenarios in which hepatocyte growth needs to be curtailed (e.g., primary liver cancers) or stimulated (e.g., fulminant liver failure). In addition, as the authors indicate, the approach can be applied to map dynamic gene expression changes in other types of liver cells, and this might identify novel therapeutic targets in liver diseases that are driven by those cells (e.g., cirrhosis that is mediated by activated hepatic stellate cells).

The authors focus on one novel finding of their TRAP-seq analysis, i.e., that hepatocytes repopulating chronically injured liver significantly upregulate many mRNAs involved in glutathione (GSH) biosynthesis, particularly Slc7a11, a gene that encodes the cystine/glutamate exchanger (xCT). This result and many other findings were validated by bulk RNA-seq analysis of whole-liver mRNAs at time points of maximal hepatocyte replication after partial hepatectomy (PHx), a classical acute regenerative stimulus. The importance of $S l c 7 a 11 / \mathrm{xCT}$ induction for hepatocyte repopulation was then confirmed by 
manipulating hepatocyte Slc7a11 expression in mice with actively injured livers.

$\mathrm{xCT}$ exports intracellular glutamate in order to import extracellular cystine that is then converted to cysteine for synthesis of GSH, a potent antioxidant. This has potential implications for liver repair, because oxidative stress is increased in hepatocytes during the regenerative challenges imposed by $\mathrm{PHx}$ or chronic liver injury, and when antioxidant mechanisms become overwhelmed, ROS trigger a senescence program that blocks cell replication. Hence, it is plausible that induction of mechanisms that constrain oxidative stress is necessary for hepatocytes to progress through the cell cycle and repopulate the liver during injury. Interestingly, xCT is highly expressed in many types of malignant cells, where it regulates signaling by ROS to modulate a cancer stem cell-like phenotype. Suppression of xCT results in excessive ROS and generally inhibits cancer cell growth by reducing cell viability and proliferation, while overexpression of $\mathrm{xCT}$ decreases ROS and promotes the acquisition of stem cell-like characteristics $(2,3)$. Evidence that both stem-like cells and regenerating hepatocytes express relatively high levels of $S l c 7 a 11$ is intriguing, because the TRAP-seq analysis of regenerating hepatocytes suggests that replicating hepatocytes are relatively immature, expressing significantly lower levels of mature hepatocyte genes (e.g., albumin, transthyretin, cytochrome P450 2E1, and asialoglycoprotein receptor) than do quiescent hepatocytes in healthy adult livers.

\section{TRAP-seq provides answers and raises further questions}

By assaying for transposase-accessible chromatin using sequencing (ATAC-seq), Wang et al. identified enhanced chromatin accessibility that permits activating transcription factor 4 (ATF4) to bind to, and transcriptionally activate, the promoter of Slc7a11 as a mechanism for induction of $\mathrm{xCT}$ in repopulating hepatocytes. Interestingly, ATF4 was identified as the most interactive component of the hepatoblast-specific transcription factor network by a recent single-cell RNA-sequencing
(scRNA-seq) analysis of mouse fetal liver cells (4), and chromatin accessibility alterations and epigenomic reprogramming are known to regulate cell plasticity, including lineage specification and pluripotency $(5,6)$. Hence, this finding supports other data from Wang et al. indicating that regenerating hepatocytes are relatively immature. Together, these observations raise interesting questions regarding how the global chromatin landscape changes during liver regeneration. Do regenerating adult hepatocytes only modify the regulatory elements of a handful of genes that perform specific regenerative functions, or do they cause a more global change that leads to a distinct epigenetic state, which may indicate a more primitive, progenitor-like state? Global analysis of the authors' ATAC-seq data may provide insights into this important question. This can be further addressed by additional epigenetic profiling approaches (e.g., bisulfite sequencing and ChIP-seq) that provide information about DNA methylation and histone modification status. The recent advancement of such technologies for profiling samples with low cell counts is making these approaches a reality for studying primary liver cells.

\section{Future directions}

Finally, regenerating livers need to replenish other cell types besides hepatocytes (e.g., cholangiocytes and stellate cells). Are those cells regenerated independently via entirely separate populations? Or is there a possibility that some regenerating hepatocytes dedifferentiate into a more multipotent state that can give rise to multiple lineages? Alternatively, do adult livers harbor populations of primitive cells that may be induced to initiate hepatocytic differentiation via cell division, as occurs with fetal liver cells (4)? Comparing the authors' ATAC-seq data with those of liver cell lineages may provide some clues. scRNA-seq has been performed to profile different fetal and adult liver cell types in an unbiased way $(4,7)$. In embryonic livers, this approach identified a fetal liver cell subpopulation that coexpresses epithelial and mesenchymal markers, suggesting that fate decisions in some immature liver cells may involve epithelial-mesenchymal transitions, as occurs during the differentiation of human embryonic stem cells into hepatocytes (4). scRNA-seq analyses were also able to delineate unsuspected spatial heterogeneity in quiescent adult livers when combined with single-molecule RNA-FISH (7), revealing the complexity of signals that modulate liver cell phenotypes, even in the absence of injury. It is obvious from these discussions that an array of rapidly evolving methodologies are generating novel data that may explain the adult liver's enormous regenerative capabilities and thereby identify new solutions to replenish hepatocyte populations that become depleted as a result of liver resection or injury.

\section{Acknowledgments}

This work was supported by the Duke Endowment and 5R37-AA010154 (to AMD) and NIH R35GM122465 (to XS).

Address correspondence to: Anna Mae Diehl, Snyderman Building, Suite 1073, Duke University, Durham, North Carolina 27710, USA. Phone: 919.684.2366; Email: annamae.diehl@duke.edu.

1. Wang AW, et al. TRAP-seq identifies cystine/glutamate antiporter as a driver of recovery from liver injury. J Clin Invest. 2018;128(6):2297-2309.

2. Tsuchihashi K, et al. The EGF receptor promotes the malignant potential of glioma by regulating amino acid transport system xc(-). Cancer Res. 2016;76(10):2954-2963.

3. Polewski MD, Reveron-Thornton RF, Cherryholmes GA, Marinov GK, Aboody KS. SLC7A11 overexpression in glioblastoma is associated with increased cancer stem cell-like properties. Stem Cells Dev. 2017;26(17):1236-1246.

4. Su X, et al. Single-cell RNA-Seq analysis reveals dynamic trajectories during mouse liver development. BMC Genomics. 2017;18(1):946.

5. Skrypek N, Goossens S, De Smedt E, Vandamme N, Berx G. Epithelial-to-mesenchymal transition: epigenetic reprogramming driving cellular plasticity. Trends Genet. 2017;33(12):943-959.

6. Roadmap Epigenomics Consortium, et al. Integrative analysis of 111 reference human epigenomes. Nature. 2015;518(7539):317-330.

7. Halpern KB, et al. Single-cell spatial reconstruction reveals global division of labour in the mammalian liver. Nature. 2017;542(7641):352-356 